\title{
Mechanism of quasistabilization of primordial black holes
}

\author{
R. Torres* \\ Department of Applied Physics, Universitat Politecnica de Catalunya (UPC), Avinguda Diagonal, 649-651, 08028 Barcelona, Spain
}

(Received 9 May 2013; published 17 June 2013)

\begin{abstract}
It is argued that primordial black holes with initial masses satisfying $M<10^{15} \mathrm{~g}$, instead of having explode, might currently be in a quasistable phase contributing to a tiny fraction of the measured dark matter. This statement is based on a computation of black hole evaporation in which energy conservation is taken into account that shows that the backreaction to Hawking radiation favors the quasistabilization of the black hole. The result is specifically shown for general spherically symmetric quantum black holes described by an effective metric independently of the specific framework from which it is derived. The quintessential primordial black hole is fully analyzed as an example.
\end{abstract}

DOI: 10.1103/PhysRevD.87.123514

PACS numbers: 95.35.+d, 04.70.Dy, 04.70.Bw

\section{INTRODUCTION}

Nowadays, the standard cosmological model includes as an essential ingredient dark matter (DM). DM is expected to account for about one quarter of the present critical energy density. However, its nature remains as one of the most important open problems in physics. Some of the candidates proposed in order to explain DM would signal new physics beyond the standard model of particle physics [1]. In particular, the most studied class of candidates is that of weakly interacting massive particles which many consider to constitute most of the DM in our Universe. If they existed, they could be, in principle, observed through their interaction with nuclei in underground detectors or the products of their annihilation or decay could be detected either through astronomical observations or on ground based experiments (for instance, at the Large Hadron Collider). Although thorough searches have so far provided negative results (see, for example, [2-5]), the experiments to try to find them are currently in a very exciting and critical phase [6,7].

Another DM candidate is primordial black holes (PBHs). PBHs are assumed to have been created during the first moments of our Universe from fluctuations in the density. In this epoch, the conditions were so extreme as to generate regions dense enough to create black holes (BHs) [8-11]. The existence of the fluctuations is reflected in the presence of acoustic peaks in the cosmological microwave background and is well established by observations. While some authors assume that $\mathrm{PBH}$ contribute to a fraction of the DM in the Universe, the exact magnitude of the contribution is not clear yet [12]. Observations constrain the amount of PBHs with current masses bigger than $10^{16} \mathrm{~g}$ (see, for example, [13] for a recent summary). The amount of PBHs that were created with initial masses bigger than $10^{9} \mathrm{~g}$ are also constrained due to the indirect effect that they would have had on nucleosynthesis and on the extragalactic photon background [14].

*ramon.torres-herrera@upc.edu
On the other hand, it is argued that $\mathrm{PBH}$ which formed with masses smaller than $10^{15} \mathrm{~g}$ cannot exist in the current Universe so that they would not contribute to dark matter. Such claim comes from the celebrated works by Hawking $[15,16]$ that showed that black holes radiate a thermal spectrum of particles. Since the black hole temperature was proportional to the inverse of the black hole mass, if one followed the evolution of the black hole as it emits radiation with its consequent mass loss, one would expect a final event in which the temperature and the luminosity would diverge, indicating a final black hole explosion. Specifically, in this approach PBHs with initial masses below around $10^{15} \mathrm{~g}$ would have evaporated in less than the age of the Universe [15].

Of course, as stated by Hawking $[15,16]$ in his pioneering works, this initial picture of black hole evaporation was incomplete. For instance, it lacked of the quantum gravity corrections that one expects to appear as the last stages of black hole evaporation are reached. Moreover, the picture was based on quantum field theory on a fixed curved background (Schwarzschild's solution) what is not in agreement with energy conservation. I.e., the energy radiated by the black hole should be balanced by a corresponding decrease of its mass, but this is unfeasible with a fixed background.

Our aim in this paper is to analyze the contribution that PBHs with initial masses smaller than $10^{15} \mathrm{~g}$ might have to the current observed amount of DM. This possibility is based on the effect that energy conservation could have in the last stages of black hole evaporation, something that is currently possible to evaluate thanks to the derivation of Hawking radiation by Parikh and Wilczek [17]. We will argue that energy conservation might be sufficient to prevent the total evaporation of black holes and that, instead, black holes could enter a quasistable phase. The approach will be rather general since a definitive quantum gravity theory is not known. In this way, the proof will be carried out for general effective spherically symmetric spacetimes describing a black hole, so that the results will be directly applicable for effective spacetimes coming from many different frameworks. 
The paper has been divided as follows. In Sec. II, we introduce the effective metric and analyze the properties that it should have in order to describe a quantum black hole. Then, Hawking radiation from the $\mathrm{BH}$ will be studied from a strict thermal point of view. This will allow us to model the thermal evaporation of the black hole and to analyze its possibilities for a total evaporation. Then, in Sec. III, we will study the black hole under the approach of energy conservation. This will lead us to a different black hole luminosity that will later be used to model the evaporation process under the requirement of energy conservation. The subsequent $\mathrm{BH}$ evolution will be then contrasted with the thermal evolution found in the previous section. The case of a PBH with a Schwarzschild background embedded in the Universe, originally treated by Hawking, will be studied in detail under the energy conservation approach in Sec. IV, as a particular example. Finally, the results are discussed in Sec. V.

Unless otherwise stated throughout this paper, we will use Planck's units in which $G=\hbar=c=k_{B}=1$.

\section{GENERAL QUANTUM BLACK HOLE AND THERMAL APPROACH}

Let us consider an isolated spherically symmetric black hole embedded in an effective asymptotically flat spacetime and which is completely characterized by its mass. The effective metric for the spherically symmetric quantum vacuum (once an induced geometry, if necessary, has been found) can be locally written without any loss of generality as

$d s^{2}=-A(R ; M) d t_{S}^{2}+f(R ; M)^{-1} d R^{2}+R^{2} d \Omega^{2}$,

where the dependence in $M$ of $A(R ; M)$ and $f(R ; M)$ stresses the fact that for every Arnowitt-Deser-Misner mass $M$ there is a specific effective metric. ${ }^{1}$ We assume that $A(R ; M)$ and $f(R ; M)$ satisfy $A(R ; M) \cdot f(R ; M) \geq 0$ in order for the geometry to be Lorentzian.

The static spacetime possesses a timelike killing vector field $\vec{v}=\partial / \partial t$ for $A>0$, so that provided that $A(R ; M)=0$ had a solution $R=R_{0}(M)$ the effective spacetime would have a killing horizon at $R_{0}$. Then, as result of, for instance, a standard Euclidean continuation of the fixed static geometry through $R_{0}$, one gets that the killing horizon emits Hawking radiation with a thermal distribution of temperature

$$
T=\frac{\kappa}{2 \pi},
$$

where $\kappa$ is the surface gravity [23] corresponding to the killing horizon

$$
\left.\kappa=\frac{1}{2} \sqrt{\frac{f}{A}} \frac{d A}{d R}\right\rfloor_{R=R_{0}} .
$$

\footnotetext{
${ }^{1}$ The reader can find numerous particular examples of this type of metric in the literature, from the (classical) Schwarzschild solution to different approaches to quantum gravity [18-22] and so on.
}

If we assumed that a black hole can be at absolute zero, then there would not be Hawking radiation and we would be dealing with a stable remnant. Indeed, these remnants have been postulated to exist and it has been argued that they could represent an important fraction of DM (see, for instance, [11,24]). However, the mechanism why they should exist is not clear ${ }^{2}$ and stable remnants are the subject of criticism (see the works in [25]). In this paper, we will not treat this possibility. Instead, we will assume that the black hole cannot be at absolute zero (or, in other words, the effective geometry is such that $\kappa \neq 0$ ). If we also require the absence of scalar curvature singularities at $R=R_{0}>0$ then we get [26] that both $A$ and $f$ should have a simple zero at $R=R_{0}$.

In this way $R_{0}$ is a killing horizon and an apparent threehorizon. $^{3}$

\section{A. Thermal flux of radiation}

If $f$ and $A$ have a simple zero at the horizon $R=R_{0}$, then, using l'Hôpital's rule, the surface gravity (2.3) can be rewritten as

$$
\left.\kappa=\frac{1}{2} \sqrt{A^{\prime} \cdot f^{\prime}}\right\rfloor_{R=R_{0}},
$$

where the primes denote derivative with respect to $R$.

In the thermal approach, a BH possessing a horizon with its corresponding non-null temperature will emit photons with a standard Boltzmann distribution,

$$
\langle n(E)\rangle_{\mathrm{Stand}}=\frac{1}{\exp (E / T)-1},
$$

where $E$ is the photon energy. Moreover, the total flux of radiated energy [27] will be given approximately by

$$
L_{\text {Stand }} \simeq \frac{1}{2 \pi} \int_{0}^{\infty}\langle n(E)\rangle_{\text {Stand }} \gamma_{E 0} E d E,
$$

where $\gamma_{E 0}$ is the greybody factor that takes into account the fraction of the radiation that does not escape to infinity but is backscattered and its subindex " 0 " indicates that here we only consider the main contribution to the greybody factor that comes from the zero angular momentum $l=0$ [28]. It can be shown (see, for example, [28]) that (without taking into account energy conservation) for any static spherically symmetric black hole with outer horizon $R_{0}$, and whenever $E M \ll 1$, the greybody factor takes the form ${ }^{4}$

\footnotetext{
${ }^{2}$ For example, it has been used the heuristic argument that a generalized uncertainty principle may prevent a black hole from evaporating completely [24].

${ }^{3}$ I.e., the expansion of the outgoing radial null geodesics will be zero at $R=R_{0}$.

${ }^{4}$ Note that this expression is only the usual simplifying approximation [27,28] valid for massless scalar particles with $E M \ll 1$, but one expects case-dependent deviations $(\sim 60 \%)$ when the greybody factor is carefully (numerically) computed [29].
} 


$$
\gamma_{E 0}=4 E^{2} R_{0}^{2}
$$

Therefore, the luminosity in the thermal approach can be approximately expressed as

$$
L_{\text {Stand }} \simeq \frac{1}{2 \pi} \int_{0}^{\infty} \frac{4 E^{3} R_{0}^{2}}{\exp (2 \pi E / \kappa)-1} d E=\frac{1}{120 \pi} R_{0}^{2} \kappa^{4} .
$$

\section{B. Modeling black hole evaporation}

In order to model the backreaction produced by the emission of radiation, let us first express the effective metric (2.1) in terms of ingoing Eddington-Finkelsteinlike coordinates $\{u, R, \theta, \varphi\}$, where the new lightlike coordinate is obtained through

$$
u=t_{S}+\int^{R} \frac{d R^{\prime}}{\sqrt{A\left(R^{\prime} ; M\right) \cdot f\left(R^{\prime} ; M\right)}} .
$$

The metric now takes the form

$d s^{2}=-A(R ; M) d u^{2}+2 \sqrt{\frac{A(R ; M)}{f(R ; M)}} d u d R+R^{2} d \Omega^{2}$.

We can model the mass lost taking into account that whenever a pair of virtual particles is created, when the particle with positive energy escapes to infinity its companion, with negative energy, falls into the black hole thus reducing the $\mathrm{BH}$ mass. Therefore, if we consider negative energy massless particles following ingoing null geodesics $u=$ constant, the mass at infinity of the black hole becomes a decreasing function $M(u)$. The metric which incorporates the effect of the decreasing $\mathrm{BH}$ mass due to the ingoing null radiation is (2.9) with $A(R ; M)$ and $f(R ; M)$ replaced by $A(R ; M(u))$ and $f(R ; M(u))$, respectively ${ }^{5}$

$d s^{2}=-A(R ; M(u)) d u^{2}+2 \sqrt{\frac{A(R ; M(u))}{f(R ; M(u))}} d u d R+R^{2} d \Omega^{2}$.

On the other hand, the flux of negative energy particles directed towards the black hole equals the flux of outgoing radiated particles that reach the future lightlike infinity and, therefore,

$$
\frac{d M}{d u}=-L_{\text {Stand }}(M)
$$

\footnotetext{
${ }^{5}$ Note that the complete effective solution should also include the outgoing radiation. However, currently there is not an elegant procedure to do this and the backreaction (mass loss) is already satisfactorily incorporated taking into account the ingoing null radiation.
}

\section{Total evaporation under the thermal approach}

Our assumption on the existence of a horizon with nonzero surface gravity together with the expression of the flux of radiation (2.8) inform us that an evaporating black hole has to emit radiation as long as it has mass. On the other hand, close to zero mass (no $\mathrm{BH}$ ), the outer horizon $R_{0}(M)$ has to approach zero. The behavior of the $\mathrm{BH}$ near zero mass depends on the behavior of $\kappa$ which, at the same time, depends on the specific form of the effective geometry near zero mass. In this way, we have the following:

Proposition 1.-Let us consider an effective metric for a black hole satisfying close to zero mass,

$$
R_{0}(M) \simeq \rho M^{\sigma}, \quad A^{\prime}\left(R_{0} ; M\right) \simeq \alpha M^{\omega}, \quad f^{\prime}\left(R_{0} ; M\right) \simeq \varphi M^{\phi},
$$

where $\sigma>0$. A thermally evaporating black hole modeled with this metric will reach the total evaporation in a finite time if, and only if, $\omega+\phi+\sigma<1 / 2$.

First, note that the requirement $\sigma>0$ comes from the fact that the horizon has to approach a null value as the $\mathrm{BH}$ completely evaporates. Now, to show the proposition we can use (2.12) in (2.4) to directly get the surface gravity for the black hole with mass close to its zero value as

$$
\kappa \simeq \sqrt{\alpha \varphi} M^{(\omega+\phi) / 2} .
$$

Using this in (2.8) in order to evaluate the mass evolution (2.11) it is easy to see that, indeed, only if $\omega+\phi+\sigma<$ $1 / 2$ zero mass is reached in a finite time. On the other hand, this together with the condition $\sigma>0$ implies that a necessary condition for the total evaporation to occur is $\omega+\phi<1 / 2$.

One can further consider the subcase in which this evaporation is explosive, meaning that the luminosity approaches infinity as the mass tends to zero.

Corollary 1.-For a thermally evaporating black hole satisfying (2.12) with $\omega+\phi+\sigma<0$ the evaporation ends in a final explosion.

What comes directly from the luminosity (2.8). Note that this together with the condition $\sigma>0$ imply that the necessary condition for the explosion to occur is $\omega+\phi<0$.

It is interesting to remark that the ultimate responsible for the explosive behavior in the thermal approach is the absence of a cutoff, which allows the emission of particles with arbitrary high energy. A particular example of this explosive behavior was obtained by Hawking in its pioneering work with a Schwarzschild background [15] (a case that will be fully analyzed in Sec. IV).

\section{BLACK HOLE EVAPORATION UNDER THE ENERGY CONSERVATION APPROACH}

Let us now consider Hawking radiation taking into account the consequences of energy conservation. As in the previous section, first we will (re)consider the static case and then we will model the backreaction. Here we will 
follow the original procedure in $[17]^{6}$ which requires to rewrite the effective metric (2.1) in Painlevé-like coordinates [33] so as to have coordinates which are not singular at the horizon. To that end, we will have to introduce a new coordinate $t$ replacing the Schwarzschild-like time $t_{S}$ such that $t=t_{S}+h(R)$, where $h(R)$ is fixed by demanding the constant time slices to be flat. In this way, one gets

$$
\begin{aligned}
d s^{2}= & -A(R ; M) d t^{2}+2 \sqrt{\frac{A(R ; M)}{f(R ; M)}(1-f(R ; M))} d t d R \\
& +d R^{2}+R^{2} d \Omega^{2},
\end{aligned}
$$

In these coordinates the radial null geodesics describing the evolution of test massless particles are given by

$$
\frac{d R}{d t}=\sqrt{\frac{A(R ; M)}{f(R ; M)}}( \pm 1-\sqrt{1-f(R ; M)}),
$$

with the upper (lower) sign corresponding to outgoing (ingoing, respectively) geodesics.

In $[17,34,35]$, it was found that, when a self-gravitating shell of energy $E$ travels in a spacetime characterized by an Arnowitt-Deser-Misner mass $M$, the geometry outside the shell is also characterized by $M$, but energy conservation implies that the geometry inside the shell is characterized by $M-E$. Moreover, the shell then moves on the geodesics given by the interior line element. In this way, according to (3.2), one expects a shell of energy $E$ to satisfy the evolution equation

$\frac{d R}{d t}=\sqrt{\frac{A(R ; M-E)}{f(R ; M-E)}}( \pm 1-\sqrt{1-f(R ; M-E)})$.

Let us now consider pair production occurring just beneath the event horizon with a positive energy particle tunneling out. The standard results of the WentzelKramers-Brillouin method for the tunneling through a potential barrier that is classically forbidden can be directly applied due to the infinite redshift near the horizon [17]. In particular, the semiclassical emission rate will be given by $\Gamma \sim \exp \{-2 \operatorname{Im} S\}$, where $S$ is the particle action. Therefore, we have to compute the imaginary part of the action for an outgoing positive energy particle which crosses the horizon $R_{0}$ outwards from $R_{\text {in }}$ to $R_{\text {out }}$,

$$
\operatorname{Im} S=\operatorname{Im} \int_{R_{\text {in }}}^{R_{\text {out }}} p_{R} d R=\operatorname{Im} \int_{R_{\text {in }}}^{R_{\text {out }}} \int_{0}^{p_{R}} d p_{R}^{\prime} d R,
$$

where we have taken into account that only $p_{R}$ (the $R$ component of the four-momentum) contributes to the imaginary part of the action. Using Hamilton's equation

\footnotetext{
${ }^{6}$ Formally, the procedure in [17] can be criticized since it lacks of an explicit invariant form [30]. However, it has been shown that invariant rewritings of the procedure do exist (see, for instance, [31,32] and references therein). In this way, for practical purposes one is fully justified to follow [17].
}

$\left.\dot{R}=+d H / d p_{R}\right\rfloor_{R}, H=M-E^{\prime}$ and with the help of (3.3) this can be written as

$$
\begin{aligned}
\operatorname{Im} S= & \operatorname{Im} \int_{M}^{M-E} \int_{R_{\text {in }}}^{R_{\text {out }}} \frac{d R}{\dot{R}} d H \\
= & \operatorname{Im} \int_{0}^{E} \int_{R_{\text {in }}}^{R_{\text {out }}} \sqrt{\frac{f\left(R ; M-E^{\prime}\right)}{A\left(R ; M-E^{\prime}\right)}} \frac{d R}{1-\sqrt{1-f\left(R ; M-E^{\prime}\right)}} \\
& \times\left(-d E^{\prime}\right) .
\end{aligned}
$$

Then, by deforming the contour of integration so as to ensure that positive energy solutions decay in time, considering that in this case

$$
\lim _{R \rightarrow R_{0}} \frac{f\left(R ; M-E^{\prime}\right)}{A\left(R ; M-E^{\prime}\right)}=\frac{f^{\prime}\left(R_{0} ; M-E^{\prime}\right)}{A^{\prime}\left(R_{0} ; M-E^{\prime}\right)},
$$

and taking into account that a particle just inside the horizon tunnels just outside a shrunken horizon $\left(R_{\text {in }}>\right.$ $R_{\text {out }}$, one gets

$$
\begin{gathered}
\int_{R_{\text {in }}}^{R_{\text {out }}} \sqrt{\frac{f\left(R ; M-E^{\prime}\right)}{A\left(R ; M-E^{\prime}\right)} \frac{d R}{1-\sqrt{1-f\left(R ; M-E^{\prime}\right)}}} \\
=-i \frac{2 \pi}{\sqrt{A^{\prime}\left(R_{0} ; M-E^{\prime}\right) f^{\prime}\left(R_{0} ; M-E^{\prime}\right)}} .
\end{gathered}
$$

Using this, we can now write (3.5) as

$$
\operatorname{Im} S=2 \pi \int_{0}^{E} \frac{d E^{\prime}}{\sqrt{A^{\prime}\left(R_{0} ; M-E^{\prime}\right) f^{\prime}\left(R_{0} ; M-E^{\prime}\right)}} .
$$

Tunneling also happens when a pair is created outside the horizon and the negative energy particle tunnels into the black hole. Then, following the procedure for the Schwarzschild case in [17], the imaginary part of the action for this ingoing particle satisfies

$$
\begin{aligned}
\operatorname{Im} & \int_{0}^{-E} \int_{R_{\text {out }}}^{R_{\text {in }}} \sqrt{\frac{f\left(R ; M-E^{\prime}\right)}{A\left(R ; M-E^{\prime}\right)}} \frac{d R}{-1+\sqrt{1-f\left(R ; M-E^{\prime}\right)}} d E^{\prime} \\
& =2 \pi \int_{0}^{E} \frac{d E^{\prime}}{\sqrt{A^{\prime}\left(R_{0} ; M-E^{\prime}\right) f^{\prime}\left(R_{0} ; M-E^{\prime}\right)}}
\end{aligned}
$$

what coincides with the result for the previous channel (3.6). Both channels contribute to the rate of the Hawking process, but we have seen that both contributions provide us with the same exponential term for the semiclassical rate

$$
\begin{aligned}
\Gamma & \sim e^{-2 \operatorname{Im} S} \\
& =\exp \left(-4 \pi \int_{0}^{E} \frac{d E^{\prime}}{\sqrt{A^{\prime}\left(R_{0} ; M-E^{\prime}\right) f^{\prime}\left(R_{0} ; M-E^{\prime}\right)}}\right) .
\end{aligned}
$$

In order to compare this result with the result in the thermal approach, note that when quadratic terms are neglected we can develop $\operatorname{Im} S$ up to first order in $E$ as 


$$
\operatorname{Im} S \simeq \frac{2 \pi}{\sqrt{A^{\prime}\left(R_{0} ; M\right) f^{\prime}\left(R_{0} ; M\right)}} E,
$$

obtaining a thermal radiation for the black hole $(\Gamma \sim \exp \{-E / T\})$ with temperature

$$
T=\frac{\sqrt{A^{\prime}\left(R_{0} ; M\right) f^{\prime}\left(R_{0} ; M\right)}}{4 \pi},
$$

that coincides with the expected temperature (2.2) computed using the surface gravity (2.4).

\section{A. Spectral distribution and luminosity}

Notwithstanding the comments about the temperature (3.9) of the black hole, it is important to remark that the higher order terms in $E$ of (3.8) imply a deviation from pure thermal emission. If we take into account energy conservation, the distribution function for the emission of photons is not the standard Boltzmann distribution (2.5), but the distribution (see [36], correcting the result in [34])

$$
\langle n(E)\rangle=\frac{1}{\exp (2 \operatorname{Im} S)-1} .
$$

For our effective metric this can be written as

$$
\langle n(E)\rangle=\frac{1}{\exp \left(4 \pi \int_{0}^{E} \frac{d E^{\prime}}{\sqrt{A^{\prime}\left(R_{0} ; M-E^{\prime}\right) f^{\prime}\left(R_{0} ; M-E^{\prime}\right)}}\right)-1} .
$$

Now, we would like to use this distribution in order to write the luminosity when energy conservation is taken into account. However, this requires the use of a greybody factor that should take energy conservation into consideration. The correct greybody factor for the effective geometry can be found directly using (2.7) and takes the form (see [37])

$$
\gamma_{\mathrm{EC}}=4 E^{2} R_{0}^{2}(M-E),
$$

where $R_{0}(M-E)$ is $R_{0}$ for a mass at infinity $M-E$.

We can use (3.10) and the greybody factor $\gamma_{\mathrm{EC}}$ in order to write the approximate luminosity when energy conservation is satisfied as

$$
\begin{aligned}
L_{\mathrm{EC}} & \simeq \frac{1}{2 \pi} \int_{0}^{M}\langle n(E)\rangle \gamma_{\mathrm{EC}} E d E \\
& =\frac{1}{2 \pi} \int_{0}^{M} \frac{4 E^{3} R_{0}^{2}(M-E)}{\exp \left(4 \pi \int_{0}^{E} \frac{d E^{\prime}}{\sqrt{A^{\prime}\left(R_{0} ; M-E^{\prime}\right) f^{\prime}\left(R_{0} ; M-E^{\prime}\right)}}\right)-1} d E,
\end{aligned}
$$

where energy conservation is also imposed in the integration limits by forbidding the emitted quantum to carry more energy than the black hole mass, i.e., $0<E \leq M$.

\section{B. Preventing total evaporation in the energy conservation approach}

In Sec. II C we saw that the thermal approach implies the total evaporation of black holes under the condition $\omega+$ $\phi+\sigma<1 / 2$. However, if these evaporating black holes are considered under the perspective of the energy conservation approach we have the following.

Proposition 2.- Let us consider an effective metric for a black hole satisfying close to zero mass

$R_{0} \simeq \rho M^{\sigma}, \quad A^{\prime}\left(R_{0} ; M\right)=\alpha M^{\omega}, \quad f^{\prime}\left(R_{0} ; M\right)=\varphi M^{\phi}$,

where $\sigma>0$ and $\omega+\phi+\sigma<1 / 2$. A black hole satisfying energy conservation and modeled with this metric will not reach total evaporation if $\omega+\phi+4 \sigma \geq-4$.

In order to show the proposition, it suffices to note that, when energy conservation is taken into account, one gets [from (3.12)] a luminosity close to zero mass

$$
L_{\mathrm{EC}} \simeq c M^{3+\frac{\omega+\phi}{2}+2 \sigma},
$$

where $c$ is a constant (see the Appendix). The solutions of the evolution equation

$$
\frac{d M}{d u}=-L_{\mathrm{EC}}
$$

for the luminosity (3.14) with initial mass $M(u=0)=$ $M_{0}>0$ satisfying $M_{0} \sim 0$, are

$$
M(u) \simeq \frac{1}{\left(M_{0}^{-\eta}+c \eta u\right)^{\frac{1}{\eta}}} \quad \text { and } \quad M(u) \simeq \frac{M_{0}}{\exp (c u)}
$$

for $\eta>0$ and $\eta=0$, respectively, where $\eta \equiv(\omega+\phi) /$ $2+2 \sigma+2$. It is now apparent that, if $\omega+\phi+4 \sigma \geq$ $-4 \Leftrightarrow \eta \geq 0$, the solutions only reach zero for $u \rightarrow \infty$.

If we compare this result with the result in the thermal approach (proposition 1) we see that the possibility of a total evaporation has been drastically reduced (see Fig. 1). In fact, one of the most surprising things about proposition 2 is that, as far as we know, their assumptions are satisfied by all effective metrics in the current literature independently of the approach to quantum gravity chosen to derive them.

Let us remark that, since the emission of particles is a probabilistic process in which discrete amounts of energy (quanta) are emitted, the continuous evolution obtained through (3.15) should be seen as just describing the mean evolution of black holes. In this way, the results can be interpreted as pointing towards an infinite mean lifetime of black holes. In other words, black holes become quasistable. 


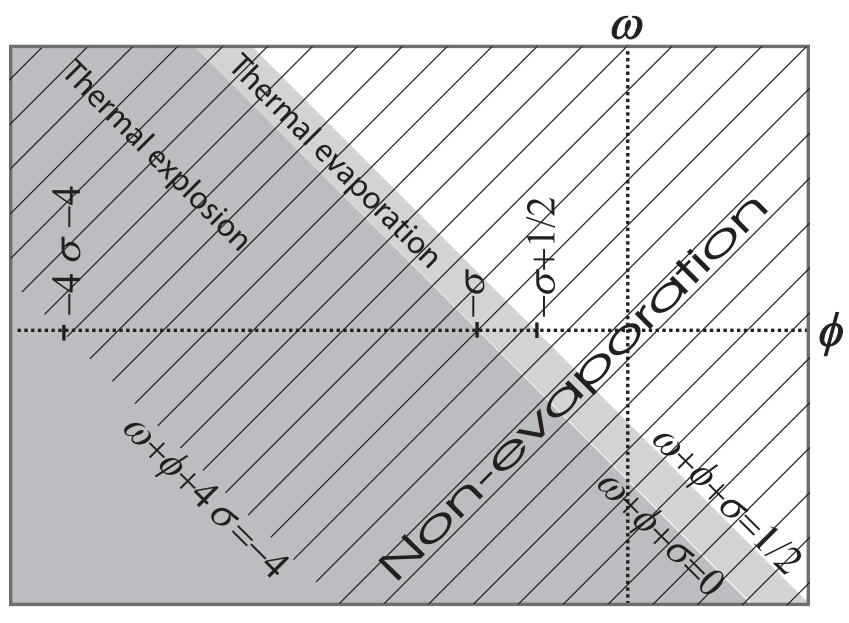

FIG. 1. Given a fixed value for $\sigma$ a schematic representation of the different possibilities in the plane $\{\phi, \omega\}$ are shown. Specifically, the darkest region correspond to the values of the exponents that allow black hole explosions in the thermal approach. The grey region close to it is the region where the thermal approach predicts total evaporation without explosion. On the other hand, the striped region (partially overlapping the previous regions) represents the region where the energy conservation approach predicts the absence of total evaporation.

\section{PBHS: THE QUINTESSENTIAL EXAMPLE UNDER THE ENERGY CONSERVATION APPROACH}

Now, we would like to consider the case of primordial black holes in a cosmological context. In general, the modeling of a black hole embedded in an evolving Universe would be too difficult were it not for some simplifying facts and assumptions. For instance, according to the work of Carr and Hawking [9], a primordial black hole will not substantially increase its original mass by accretion. Moreover, for the case of tiny PBHs their cross sections are so small that accretion can be totally neglected. In this way, PBHs could be modeled, for example, as isolated objects surrounded by the Universe in an Einstein-Strauss swiss-cheese-like model [38-40]. In other words, (2.10) with $R \ll R_{\mathrm{CH}}$ (where $R_{\mathrm{CH}}$ is the radius of the cosmological horizon) corresponds to the effective geometry close enough to the black hole. Therefore, (2.10) is the proper key ingredient in order to compute Hawking radiation from a $\mathrm{PBH}$.

Following this approach, let us now consider the standard case which gave rise to the usual assumption in the current literature about primordial black holes consisting in stating that those possessing an initial mass satisfying $M \leq 10^{15} \mathrm{~g}$ would have already evaporated. I.e., let us now treat the case of the standard evaporation of a Schwarzschild black hole. We have

$$
A(R ; M)=f(R ; M)=1-\frac{2 M}{R} ; \quad R_{0}=2 M,
$$

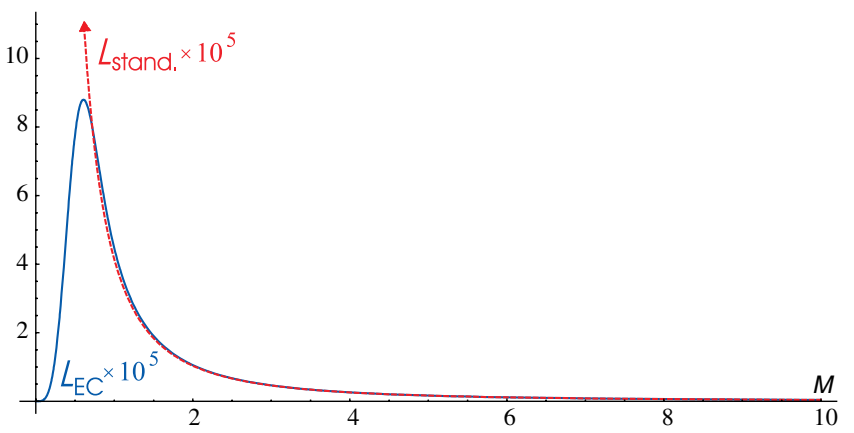

FIG. 2 (color online). The luminosity of a black hole as a function of its mass (taking into account backscattering) in Planck's units. Energy conservation has been imposed in order to draw the luminosity $L_{\mathrm{EC}}$ (solid line), while the luminosity $L_{\text {Stand }}$ (dashed line) neglects energy conservation. As can be seen, by considering energy conservation the luminosity reaches a maximum for a non-null mass. If energy conservation is neglected, the luminosity diverges as the black hole approaches its total evaporation.

so that $\omega_{\mathrm{Sch}}=\phi_{\mathrm{Sch}}=-1$ and $\sigma_{\mathrm{Sch}}=1$. Since $\omega_{\mathrm{Sch}}+$ $\phi_{\mathrm{Sch}}+\sigma_{\mathrm{Sch}}=-1$, proposition 1 implies that, according to the thermal picture, the $\mathrm{BH}$ should evaporate completely. On the other hand, corollary 1 implies the wellknown result [15] that the total evaporation in this case is accompanied by an explosion. In effect, from (2.8) one gets

$$
L_{\text {Stand }}=\frac{1}{7680 \pi M^{2}},
$$

so that the luminosity diverges as the mass approaches zero. Moreover, solving (2.11) one can get that the evaporation time ${ }^{7}$ in this case (for an initial mass $M(u=0)=$ $\left.M_{0}\right)$ is $u_{\text {Evap }}=2560 \pi M_{0}^{3}$.

However, under the energy conservation picture and since $\omega_{\text {Sch }}+\phi_{\text {Sch }}+4 \sigma_{\text {Sch }}=2>-4$, proposition 2 informs us that the total evaporation is never reached. In this case, the luminosity can be computed for all the range of masses using (3.12) as

$L_{\mathrm{EC}}(M) \simeq \frac{1}{2 \pi} \int_{0}^{M} \frac{16 E^{3}(M-E)^{2}}{\exp \{8 \pi E(M-E / 2)\}-1} d E$.

The behavior of the luminosity when energy conservation is satisfied is shown in Fig. 2 for masses satisfying $M \leq$ 10 , where it is also compared with the thermal result. In general, for macroscopic black holes the luminosities are practically identical regardless of whether energy conservation is taken into account (Eq. (4.2)) or not (Eq. (4.1)). ${ }^{8}$ For these black holes, the luminosity should grow as their

\footnotetext{
${ }^{7}$ Of course, this is the usual approximate evaporation time when one includes backscattering [27].

${ }^{8}$ However, the luminosity when energy conservation is considered is barely bigger than in the standard result all the way from macroscopic masses to masses just slightly above the maximum of luminosity.
} 

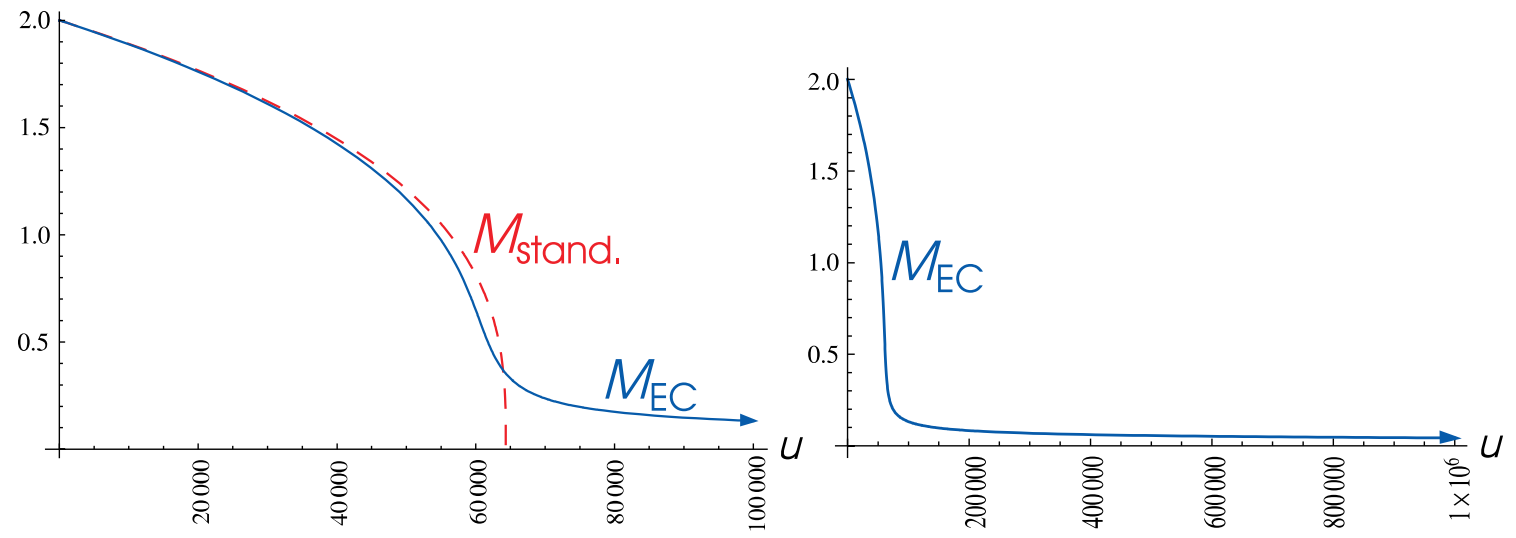

FIG. 3 (color online). To the left, we compare the evolution of a black hole mass without taking into account energy conservation $M_{\text {Stand }}(u)$ (dashed line) and taking it into account $M_{\mathrm{EC}}(u)$ (solid line) in both cases starting with the same initial mass of 2 Planck masses at $u=0$. The formation of a slowly evaporating remnant, if energy conservation is taken into account, contrasts with the sudden total evaporation in the "thermal" approximation. To the right, we have depicted the evaporating remnant in the broader range $0 \leq u \leq 10^{6}$.

masses decrease due to the emission of particles. The differences only become important when the masses are of the order of Planck's mass (see Fig. 2). We notice that the luminosity in the thermal approach keeps increasing while, considering energy conservation, the luminosity reaches a maximum and then decreases as the black hole keeps evaporating. Let us remark that one should consider the divergent behavior of the luminosity in the thermal approach as nonsensical and due to allowing the black hole to emit particles with energies bigger than its own mass.

The evolution of the black hole can also be numerically computed for an arbitrary period of time by evaluating [from (3.15)] the integrodifferential equation

$\frac{d M(u)}{d u}=-\frac{1}{2 \pi} \int_{0}^{M(u)} \frac{16 E^{3}(M(u)-E)^{2}}{\exp \{8 \pi E(M(u)-E / 2)\}-1} d E$.

In Fig. 3, black hole evolution has been drawn satisfying energy conservation in an interval including the beginning of the quasistable phase. Starting with an initial mass $M_{0}$ greater than Planck's mass, the $\mathrm{BH}$ reaches its quasistable phase at

$$
u_{q-s}=2560 \pi M_{0}^{3},
$$

i.e., when it should have completely evaporated according to the thermal approach. Prior to this, the evaporation process is a little bit faster if energy conservation is taken into account due to its slightly higher luminosity. However, and most importantly, the evaporation process decelerates around $u_{q-s}$ when energy conservation is taken into account, what eventually leads to the formation of a quasistable $\mathrm{BH}$ as the luminosity becomes more and more dim.

Another interesting aspect of the evaporation is the effective spectral distribution of the emitted particles. The effective spectral distribution is the distribution of energies for the photons emitted from the $\mathrm{BH}$ that a distant observer sees. In the thermal approach, it is simply
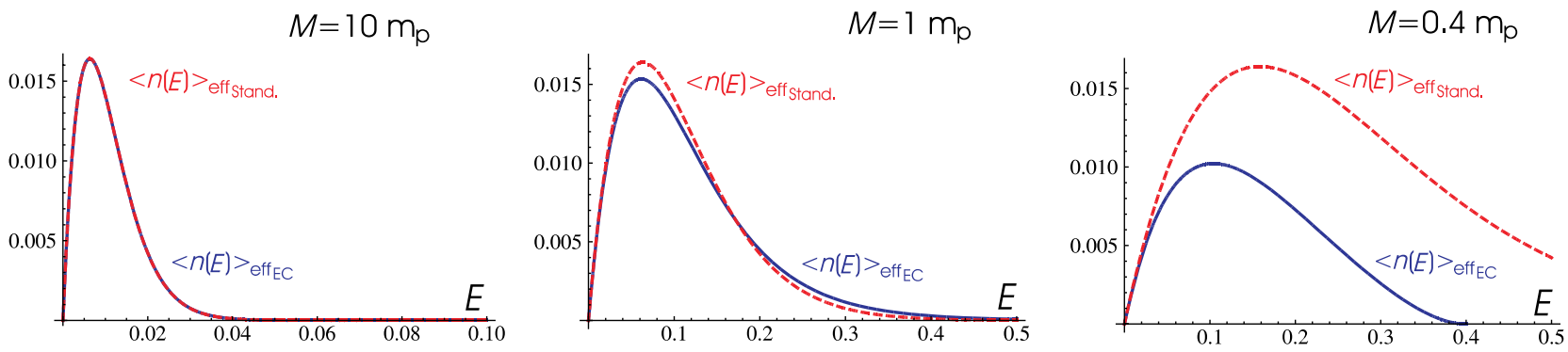

FIG. 4 (color online). A comparison of the effective spectral distributions of the radiation as seen from infinity (taking into account backscattering). Specifically, we have plotted it for three different BH masses as a function of the energy $E$ in Planck's units. Energy conservation has been imposed in order to draw the solid lines $\left(\langle n(E)\rangle_{\mathrm{Eff}_{\mathrm{EC}}}\right)$, while the dashed lines have been drawn neglecting energy conservation $\left(\langle n(E)\rangle_{\mathrm{Eff}_{\text {Stand }}}\right)$. For masses bigger that the Planck mass the curves are nearly identical (see, for instance, the first diagram with a mass corresponding to 10 Planck masses). The differences are only considerable if the mass of the black hole is around or below the Planck mass (second and third diagrams, respectively). 
$\langle n(E)\rangle_{\mathrm{Eff}_{\text {Stand }}}=\langle n(E)\rangle_{\text {Stand }} \cdot \gamma_{E 0}=\frac{16 E^{2} M^{2}}{\exp (8 \pi E M)-1}$,

while in the energy conservation approach takes the form

$$
\langle n(E)\rangle_{\mathrm{Eff}_{\mathrm{EC}}}=\langle n(E)\rangle_{\mathrm{EC}} \cdot \gamma_{\mathrm{EC}}=\frac{16 E^{2}(M-E)^{2}}{\exp \left\{8 \pi E\left(M-\frac{E}{2}\right)\right\}-1} .
$$

Both effective distributions are compared in Fig. 4. As can be expected, the effective distributions for the thermal and the energy conservation approaches are practically identical for macroscopic masses. However, they differ significantly for BH masses of the order or smaller than Planck's mass. This can be described as follows. In the thermal approach, the temperature always increases as the $\mathrm{BH}$ mass diminishes. This implies that more higher energy particles are emitted as the last stages of evaporation are reached. On the other hand, in the energy conservation approach, previous to achieving the order of Planck's mass the situation is similar. However, as the mass keeps decreasing the maximum of energy for the emitted photons starts decreasing until in most of the quasistable phase of the black hole only long wave photons are allowed to be emitted.

\section{CONCLUSIONS}

In this paper, we have seen that when energy conservation is imposed in the evaporation of black holes the possibility of total evaporation (and, of course, of a final explosion) seems to be drastically reduced (proposition 2). In fact, one can realize of the importance of this reduction by noticing that, as far as we know, the proposed effective solutions for black holes (coming from different approaches to quantum gravity) satisfy the conditions that would be required for a quasistabilization of the black hole. Of course we cannot use a definitive quantum theory of gravity in order to rigourously test this result. Instead, we speculate that energy conservation might be the sufficient physical mechanism for the quasistabilization of black holes because, contrary to the thermal approach, it avoids the emission of particles with arbitrary high energies.

It is well known that most of the PBHs should have been formed with small initial masses [10,11] (a possibility that is even favored in certain inflationary scenarios [24,41]). This fact would be considered not connected with the amount of dark matter in our current Universe under the assumption that black holes with initial masses bellow $10^{15} \mathrm{~g}$ would have already exploded. However, we have seen that, under the assumption of energy conservation, these black holes might have survived in the form of quasistable primordial black holes. In order to have a numerical idea of the contribution of quasistable PBHs to dark matter, we can use our example in Sec. IV. This is so because, even if nowadays the precise evolution of a PBH is model dependent, if the true scale of gravity were Planck's mass the numerical results would not differ very significantly from the ones that can be obtained from the standard PBH treated there. Thus, the rough numbers [from (4.3)] would be as follows. Starting, for instance, from a collection of PBHs with initial masses of around $5 m_{p}$, these black holes would have lost most of their masses and have entered its quasistable phase in around $10^{-38} \mathrm{~s}$ after their formation. From here, their emission rate would descend sharply. For example, 379 thousand years later, during the recombination epoch, their expected mass would be of around $8 \mathrm{GeV}$. Nowadays, they would be part of the light DM with an expected mass of around $200 \mathrm{MeV}$.

One can then infer that, thanks to their evaporation, quasistable PBHs would have lost most of their mass by releasing it to the rest of the Universe. Therefore, only a tiny fraction of the current dark matter could be in the form of quasistable PBHs.

With regard to the detection of quasistable black holes, as interactions with them would be purely gravitational and their cross sections are extremely small, direct observation of quasistable PBHs seems too difficult. However, the quasistabilization of black holes has clear signatures that differentiate their behavior from the one expected in the thermal picture. This could be better observed under controlled conditions. For example, assuming that black holes could be created in colliders at attainable energies (see, for example, [42] for a review), probably the best chance of observing these signatures would be after black hole creation in particle collisions [43].

\section{APPENDIX: CALCULATION OF $L_{\mathrm{EC}}$ FOR $M \sim 0$}

In order to show (3.14) for $\omega+\phi+\sigma<1 / 2(\Rightarrow \omega+$ $\phi<1 / 2)$, we have to compute, for $M \sim 0$, the result of the integral

$$
L_{\mathrm{EC}} \simeq \frac{1}{2 \pi} \int_{0}^{M} \frac{4 E^{3} R_{0}^{2}(M-E)}{\exp \left(4 \pi \int_{0}^{E} \frac{d E^{\prime}}{\sqrt{A^{\prime}\left(R_{0} ; M-E^{\prime}\right) f^{\prime}\left(R_{0} ; M-E^{\prime}\right)}}\right)-1} d E,
$$

with

$$
\sqrt{A^{\prime}\left(R_{0} ; M-E^{\prime}\right) f^{\prime}\left(R_{0} ; M-E^{\prime}\right)} \simeq \sqrt{\alpha \varphi}\left(M-E^{\prime}\right)^{(\omega+\phi) / 2} .
$$

A change of variables $\left\{x=E^{\prime} / M\right\}$ and the calculation of the corresponding integral allows us to write the argument in the exponential approximately as

$$
\epsilon \equiv \frac{4 \pi\left[1-(1-x)^{1-\Upsilon}\right]}{\sqrt{\alpha \varphi}(1-\Upsilon)} M^{1-\Upsilon},
$$

where $Y \equiv(\omega+\phi) / 2<1 / 4<1$. Since $\epsilon$ is a small quantity for $M \sim 0$, one has $e^{\epsilon}-1 \simeq \epsilon$, so that the 
luminosity close to zero mass $(M \sim 0)$ can be directly computed as

$$
L_{\mathrm{EC}} \simeq \frac{\rho^{2}(1-\Upsilon) I \sqrt{\alpha \varphi}}{2 \pi^{2}} M^{3+\Upsilon+2 \sigma},
$$

where we have used that $R_{0} \simeq \rho M^{\sigma}$ and we have defined

$$
I \equiv \int_{0}^{1} \frac{x^{3}(1-x)^{2 \sigma} d x}{1-(1-x)^{1-\Upsilon}}
$$

Undoing the change of variables, one directly obtains (3.14) with the sought constant $c$.
[1] G. Bertone, D. Hooper, and J. Silk, Phys. Rep. 405, 279 (2005).

[2] A. Geringer-Sameth and S.M. Koushiappas, Phys. Rev. Lett. 107, 241303 (2011).

[3] Ackermann et al., Phys. Rev. Lett. 107, 241302 (2011).

[4] D. B. Cline, arXiv:1209.4037.

[5] M. G. Aartsen et al. (IceCube Collaboration), Phys. Rev. Lett. 110, 131302 (2013).

[6] G. Bertone, Nature (London) 468, 389 (2010).

[7] A. Cho, Science 340, 418 (2013).

[8] Y. B. Zel'dovich and I. D. Novikov, Sov. Astron. 10, 602 (1967).

[9] B. J. Carr and S. W. Hawking, Mon. Not. R. Astron. Soc. 168, 399 (1974).

[10] B. J. Carr, Astrophys. J. 201, 1 (1975).

[11] B. J. Carr, 22nd Texas Symposium, Stanford, December, 12-17 2004, eConf C041213, 0204 (2004).

[12] M. R. S. Hawkins, Mon. Not. R. Astron. Soc. 415, 2744 (2011).

[13] F. Capela, M. Pshirkov, and P. Tinyakov, Phys. Rev. D 87, 023507 (2013).

[14] B. J. Carr, K. Kohri, Y. Sendouda, and J. Yokoyama, Phys. Rev. D 81, 104019 (2010).

[15] S. W. Hawking, Nature (London) 248, 30 (1974).

[16] S. W. Hawking, Commun. Math. Phys. 43, 199 (1975).

[17] M. K. Parikh and F. Wilczek, Phys. Rev. Lett. 85, 5042 (2000).

[18] I. G. Dymnikova, Gen. Relativ. Gravit. 24, 235 (1992); Phys. Lett. B 472, 33 (2000).

[19] G. Horowitz, J. Maldacena, and A. Strominger, Phys. Lett. B 383, 151 (1996); J. Maldacena, Adv. Theor. Math. Phys. 2, 231 (1998).

[20] L. Modesto, Int. J. Theor. Phys. 49, 1649 (2010); S. Hossenfelder, L. Modesto, and I. Prémont-Schwarz, Phys. Rev. D 81, 044036 (2010).

[21] A. Bonanno and M. Reuter, Phys. Rev. D 62, 043008 (2000).

[22] P. Nicolini, Int. J. Mod. Phys. A 24, 1229 (2009); Y. S. Myung, Y.-W. Kim, and Y.-J. Park, J. High Energy Phys. 02 (2007) 012.
[23] R. M. Wald, General Relativity (University of Chicago, Chicago, 1984).

[24] P. Chen, New Astron. Rev. 49, 233 (2005).

[25] J. Preskill, arXiv:hep-th/9209058; S. B. Giddings, Phys. Rev. D 49, 947 (1994); L. Susskind, arXiv:hep-th/ 9501106.

[26] R. Torres and F. Fayos, [Phys. Rev. D (to be published)].

[27] A. Fabbri and J. Navarro-Salas, Modeling Black Hole Evaporation (Imperial College Press, London, 2005).

[28] T. Harmark, J. Natario, and R. Schiappa, Adv. Theor. Math. Phys. 14, 727 (2010).

[29] R. Balbinot, A. Fabbri, V. P. Frolov, P. Nicolini, P. Sutton, and A. I. Zelnikov, Phys. Rev. D 63, 084023 (2001).

[30] B. D. Chowdhury, Pramana J. Phys. 70, 3 (2008).

[31] R. Di Criscienzo, S. A. Hayward, M. Nadalini, L. Vanzo, and S. Zerbini, Classical Quantum Gravity 27, 015006 (2010).

[32] L. Vanzo, G. Acquaviva, and R. Di Criscienzo, Classical Quantum Gravity 28, 183001 (2011).

[33] P. Painlevé, C. R. Acad. Sci. (Paris) 173, 677 (1921).

[34] P. Kraus and R. Wilczeck, Nucl. Phys. B433, 403 (1995); B437, 231 (1995).

[35] W. Israel and Z. Yun, Phys. Rev. D 82, 124036 (2010).

[36] E. Keski-Vakkuri and P. Kraus, Nucl. Phys. B491, 249 (1997).

[37] R. Torres, F. Fayos, and O. Lorente-Espín, Phys. Lett. B 720, 198 (2013).

[38] A. Einstein and E. G. Strauss, Rev. Mod. Phys. 17, 120 (1945).

[39] K. Lake, Astrophys. J. 240, 744 (1980); 242, 1238 (1980).

[40] F. Fayos, J. M. M. Senovilla, and R. Torres, Phys. Rev. D 54, 4862 (1996).

[41] D. Blais, C. Kiefer, and D. Polarski, Phys. Lett. B 535, 11 (2002).

[42] S. C. Park, Prog. Part. Nucl. Phys. 67, 617 (2012).

[43] R. Torres, F. Fayos, and O. Lorente-Espín (unpublished). 